Review Article

\title{
Evolocumab: rising momentum as novel antidyslipidemic drug
}

\author{
Rekha Mehani', Ajay Shukla2*, V. K. Yadav², Rimjhim Sahu' ${ }^{1}$
}

${ }^{1}$ Department of Pharmacology, RKDF Medical College Hospital and Research Center, Bhopal, Madhya Pradesh, India ${ }^{2}$ Department of Pharmacology, Gandhi Medical College, Bhopal, Madhya Pradesh, India ${ }^{3}$ Department of Pharmacology, People's College of Medical Sciences and Research Centre, Bhopal, Madhya Pradesh, India

Received: 15 April 2017

Received: 15 May 2017

Accepted: 19 May 2017

\section{*Correspondence to:}

Dr. Ajay Shukla,

Email: drajay1024@gmail.com

Copyright: (C) the author(s), publisher and licensee Medip Academy. This is an openaccess article distributed under the terms of the Creative Commons Attribution NonCommercial License, which permits unrestricted noncommercial use, distribution, and reproduction in any medium, provided the original work is properly cited.

\begin{abstract}
Increased levels of low density lipoprotein cholesterol are responsible for the major cardiovascular events. Low density lipoprotein cholesterol reduction has proved to be highly effective in reducing the risk of major cardiovascular $(\mathrm{CV})$ events in various trials. ACC/AHA guidelines recommend lipid-lowering therapy for patients with known cardiovascular diseases (CVD). Statins are the gold standard treatment for all types hypercholeterolemia but still there is need of some other lipid-lowering therapies especially in patients with statin intolerance and in patients responding inadequately to statins. Proprotein convertase subtilisin/kexin type 9 (PCSK9) was discovered in 2003 and subsequently emerged as a novel target for LDLC-lowering therapy. Evolocumab is a fully human monoclonal immunoglobulin G2 (IgG2) directed against human PCSK9. Evolocumab binds to PCSK9 enzyme rendering it unable to bind to the LDLR. More LDLR are available to bind to LDLC. Evolocumab increase the density of LDLR on the surface of hepatocytes, thereby increasing the uptake of LDL particles and decreasing the LDLC in the blood. Evolocumab has proved its efficacy with LDLC reduction from 53\% to $75 \%$ and associated with minor side effects. Evolocumab has corroborated its effectiveness in reduction in the levels of LDLC. This drug has shown efficacy in heterozygous and homozygous subtypes of familial hypercholesterolemia. Statin intolerance seen in about $15 \%$ of all patients restricts the use of first line drug for dyslipidemia. Evolocumab can be a useful option in statin intolerant patients and in patients responding inadequately to statins.
\end{abstract}

Keywords: Alirocumab, Dyslipidemia, Evolocumab, Hypercholesterolemia, Proprotein convertase subtilisin/ kexin type 9

\section{INTRODUCTION}

Low density lipoprotein cholesterol (LDLC) reduction has proved to be highly effective in reducing the risk of major cardiovascular (CV) events in various trials..$^{1-3}$ American College of Cardiology (ACC) and American Heart Association (AHA) guidelines recommend lipidlowering therapy for patients with known cardiovascular diseases (CVD)., ${ }^{4,5}$ Statins have shown, as first-line pharmacotherapy, the significant reduction in LDLC and further CV events. ${ }^{6,7}$ Despite high statin therapy, some patients cannot achieve recommended target levels of LDLC. $^{8-10}$

LDLC binds to low density protein receptor (LDLR). LDLC-LDLR complex undergoes endocytosis. Then this complex dissociates and LDLC undergoes lysosomal degradation and LDLR is released. Proprotein convertase subtilisin/kexin type 9 (PCSK9) is a serine protease protein produced in the liver, plays an important role in modulation of the LDLC receptor in multiple organs. PCSK9 binds to LDLR and causes internalization and 
degradation of LDLR. Thus LDLR is unavailable on the surface of hepatocytes. ${ }^{11}$

PCSK9 antibodies, Alirocumab and Evolocumab, bind to PCSK9 enzyme rendering it unable to bind to the LDLR. More LDLR are available to bind to the LDLC. PCSK9 antibodies increase the density of LDLR on the surface of hepatocytes, thereby increasing the uptake of LDL particles and decreasing the LDLC in the blood (Figure 1). ${ }^{12}$

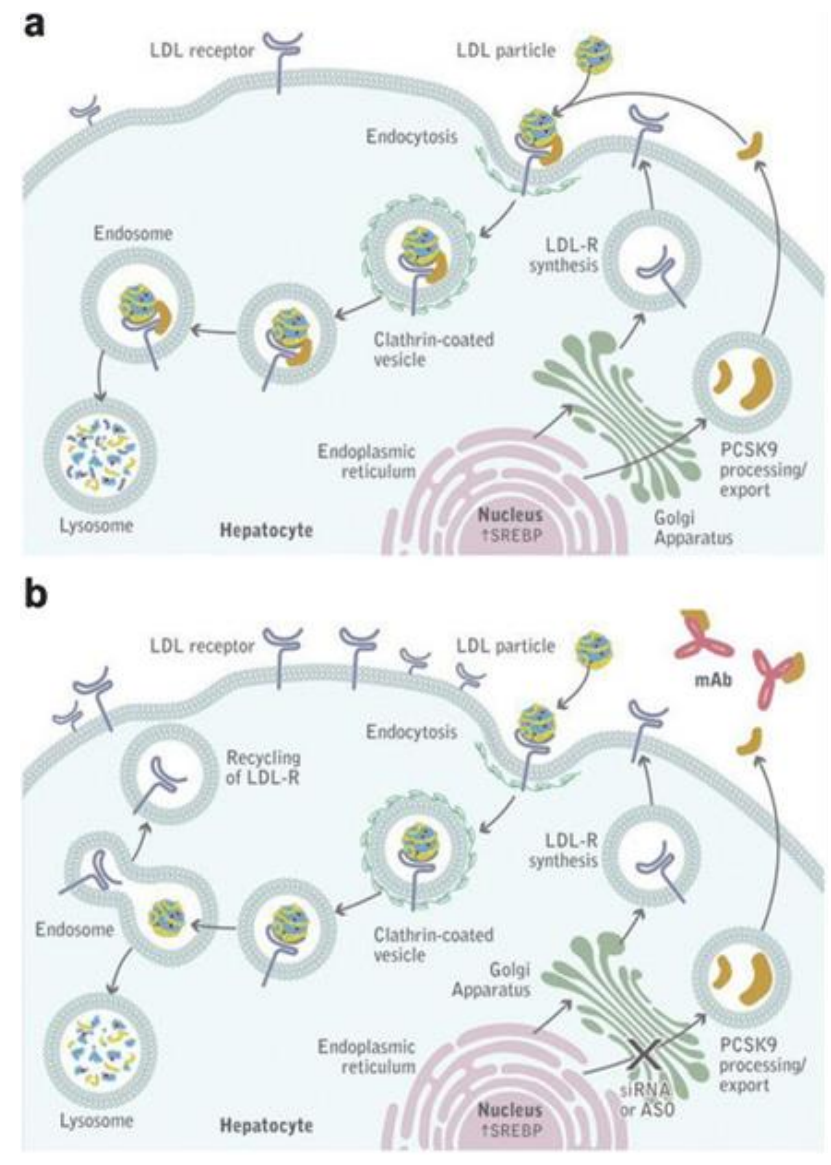

Figure 1: PCSK9 mediated degradation of LDL receptors.

Many trials have evaluated the safety and efficacy of evolocumab. These trials serve to clarify the efficacy of evolocumab in different groups of patients, and are critically reviewed here with particular reference to their clinical utility, and their place in future clinical practice. $^{13-15}$

\section{GAUSS-2 STUDY}

The Goal Achievement after Utilizing an Anti-PCSK9 Antibody in Statin Intolerant Subjects (GAUSS-2) study was a 12 weeks duration, randomized, double-blind, placebo and ezetimibe-controlled, phase III clinical trial that has been published in the Journal of the American College of Cardiology in June 2014. ${ }^{16}$ The aim of the study was to assess the efficacy and tolerability of evolocumab in patients with statin intolerance due to muscle-related side effects.

A total of 307 patients (age $62 \pm 10$ years; LDLC $193 \pm 59 \mathrm{mg} / \mathrm{dl}$ ) were randomized. Four groups were assigned. First group had received evolocumab 140mg every two weeks (Q2W) with the oral placebo, the second group had evolocumab $420 \mathrm{mg}$ once monthly (QM) with the oral placebo, the third group had subcutaneous placebo Q2W with daily oral ezetimibe $10 \mathrm{mg}$ and the fourth group had subcutaneous placebo QM with daily oral Ezetimibe $10 \mathrm{mg}$. LDLC reduced by evolocumab from baseline by $53 \%$ to $56 \%$, corresponding to treatment differences versus Ezetimibe of $37 \%$ to $39 \%$ ( $p<0.001$ ). At a mean of weeks 10 and 12, evolocumab achieved mean percent reductions of LDLC of $56.1 \%$ (Q2W dose) and $55.3 \%$ (QM dose), compared to $36.9-38.7 \%$ in Ezetimibe-treated patients $(\mathrm{p}<0.001)$. evolocumab also reduced lipoprotein (a) levels by $27 \%(\mathrm{Q} 2 \mathrm{~W})$ and $22 \%$ (QM) at week 12 .

Evolocumab was well tolerated with $96 \%$ of patients completing treatment. Incidence of myalgia was low (18\%, $7 \%$, and $9 \%$ of patients in the ezetimibe, evolocumab Q2W, and evolocumab QM groups, respectively).

\section{MENDEL-2}

MENDEL-2 was a randomized, controlled phase III clinical trial of evolocumab published in 2014. ${ }^{17}$ Aim of this study was to compare biweekly and monthly evolocumab with placebo and oral ezetimibe in patients with hypercholesterolemia.

A total of 614 patients of 18 to 80 years of age, with fasting LDLC $\geq 100$ and $<190 \mathrm{mg} / \mathrm{dl}$, were randomized (1:1:1:1:2:2) to oral placebo and subcutaneous (SC) placebo biweekly; oral placebo and SC placebo monthly; ezetimibe and SC placebo biweekly; ezetimibe and SC placebo monthly; oral placebo and evolocumab 140mg biweekly; or oral placebo and evolocumab 420mg monthly.

Evolocumab reduced LDLC from baseline, by $55 \%$ to $57 \%$ more than placebo and $38 \%$ to $40 \%$ more than Ezetimibe ( $\mathrm{p}<0.001)$. Evolocumab treatment also favorably altered other lipoprotein levels. It was well tolerated. Treatment-emergent adverse events (AEs), muscle-related AEs were comparable across all treatment groups.

\section{LAPLACE-2 STUDY}

The LDLC Assessment with PCSK9 Monoclonal Antibody Inhibition Combined with Statin Therapy (LAPLACE-2) study was a Phase III, randomized, double-blind, placebo- and ezetimibe-controlled study which was conducted in 198 sites published in the Journal of the American Medical Association in May 
2014. ${ }^{18}$ The objective of the trial was to evaluate the efficacy and tolerability of evolocumab when used in combination with a moderate-or high-intensity statin.

After a 4-week lipid-stabilization with moderate and high intensity statins, 1899 patients were randomized to compare evolocumab (140mg Q2W or 420mg QM) with placebo (Q2W or QM) or ezetimibe $(10 \mathrm{mg}$ or placebo daily; atorvastatin patients only) when added to statin therapies. Evolocumab reduced LDLC levels by $66 \%$ to $75 \%(\mathrm{Q} 2 \mathrm{~W})$ and by $63 \%$ to $75 \%(\mathrm{QM})$ vs placebo at the mean of weeks 10 and 12 in the moderate- and highintensity statin- treated groups. Adverse events were reported in evolocumab, ezetimibe and placebo-treated patients, 36\%, 40\%, and 39\% respectively. The most common adverse events in evolocumab-treated patients were muscle spasms, back pain, pain in extremities, arthralgia, and headache (all <2\%).

\section{DESCARTES STUDY}

The Durable Effect of PCSK9 Antibody Compared with Placebo Study (DESCARTES) study was a randomized, double-blind, placebo-controlled, phase III trial that was conducted at 88 centers, and was published in the New England Journal of Medicine in May 2014. ${ }^{19}$ Patients with hyperlipidemia were started on lipid lowering therapy diet alone, atorvastatin $10 \mathrm{mg}$, atorvastatin $80 \mathrm{mg}$, atorvastatin $80 \mathrm{mg}$ plus ezetimibe $10 \mathrm{mg}$ daily for 4 to 12 weeks. Those patients have cholesterol level of $>75 \mathrm{mg} / \mathrm{dl}$ are selected for study in 2:1 ratio to receive either evolocumab (420mg) or placebo for 4 weeks. Percent change from baseline in LDL cholesterol was measured at week 52. Evolocumab produced $57 \%$ mean reduction in LDLC versus placebo. In the diet-alone group, reduction in LDLC was $55.7 \%, 61.6 \%$ in the atorvastatin $10 \mathrm{mg}$ group, $56.8 \%$ in the atorvastatin $80 \mathrm{mg}$ group, and $48.5 \%$ in the atorvastatin $80 \mathrm{mg}$ plus ezetimibe $10 \mathrm{mg}$ group. Patients achieved LDLC concentration $70 \mathrm{mg} / \mathrm{dL}$ were $82.3 \%$. There were also significant reductions from baseline in apolipoprotein B, non-High-Density Lipoprotein (HDL) cholesterol, lipoprotein (a), and triglycerides, as well as $5.4 \%$ and $3.0 \%$ increases in HDLC and apo-A1.

Evolocumab treatment also significantly reduced levels of apo-lipoprotein B, non-high-density lipoprotein cholesterol, lipoprotein (a), and triglycerides. The most common adverse events were nasopharyngitis, upper respiratory tract infection and back pain. Myalgia was reported by $4 \%$ of patients on evolocumab vs. $3 \%$ on placebo.

\section{TESLA PART B STUDY}

The Trial Evaluating PCSK9 Antibody in Subjects with LDL Receptor Abnormalities (TESLA) study was a randomized, double-blind, placebo-controlled phase 3 trial was undertaken at 17 across in ten countries in North America, Europe, the Middle East, and South Africa that has been published in the Lancet journal in October $2014 .^{20}$ There were 50 eligible patients with familial hypercholesterolemia who were randomly allocated in a ratio of 2:1 to receive subcutaneous evolocumab $420 \mathrm{mg}$ or placebo every 4 weeks for 12 weeks. The primary endpoint was percent change in LDLC from baseline. These 50 enrolled patients were on stable lipid-regulating therapy for at least four weeks and were not receiving lipoprotein apheresis. Among 49 patients who completed the study, 33 patients received evolocumab 420mg and 16 patients received placebo every 4 weeks for 12 weeks.

Evolocumab significantly reduced LDL cholesterol at 12 weeks by $30.9 \%$ Compared with placebo. Treatmentemergent adverse events occurred in $12(36 \%)$ of 33 in the evolocumab group and $10(63 \%)$ of 16 patients in the placebo group. No serious clinical or laboratory adverse events occurred.

\section{RUTHERFORD-2}

This study was multicentric, randomized, double-blind, placebo-controlled, undertaken at 39 sites (most of which were specialized lipid clinics) in Australia, Asia, Europe, New Zealand, North America, and South Africa published in the Lancet journal in January 2015. ${ }^{22}$ The objective of this study was to assess safety and efficacy of evolocumab in patients with heterozygous familial hypercholesterolemia and LDLC $>100 \mathrm{mg} / \mathrm{dl}$ despite of ongoing lipid-lowering therapy.

A total of 331 patients of $18-80$ years age, were randomly allocated in a 2:2:1:1 ratio to receive subcutaneous evolocumab 140mg Q2W, evolocumab 420mg QM, or subcutaneous placebo Q2W or QM for 12 weeks. Evolocumab at both dosing schedules led to a significant reduction in mean LDLC at week 12 (Q2W dose: $59.2 \%$ reduction, QM dose: $61.3 \%$ reduction; both $\mathrm{p}<0.0001)$ and at the mean of weeks 10 and $12(60 \cdot 2 \%$ reduction and $65.6 \%$ reduction; both $\mathrm{p}<0.0001)$. Adverse events caused by evolocumab were similar to placebo. Nasopharyngitis and muscle-related adverse events were $9 \%$ vs $5 \%$ and $5 \%$ vs $1 \%$ in evolocumab and placebo group respectively.

\section{OSLER-1 AND OSLER-2}

Open-Label Study of Long-Term Evaluation against LDL Cholesterol (OSLER) trials were open-label, randomized, controlled study conducted at 305 centers that participated in at least one of seven phase 3 studies of evolocumab. ${ }^{16-19,21-24} \mathrm{~A}$ total of 4465 patients were randomly assigned in a 2:1 ratio to receive either evolocumab (140mg Q2W or 420mg QM) plus standard therapy or standard therapy alone. Evolocumab reduced the LDL cholesterol level by $61 \%$ (95\% confidence interval [CI], 59 to $63 ; \mathrm{p}<0.001)$, reductions of $52.0 \%$ in non-HDL cholesterol, $47.3 \%$ in apolipoprotein B, $36.1 \%$ in total cholesterol, $12.6 \%$ in triglycerides, and $25.5 \%$ in lipoprotein (a). 
Most of the adverse events were similar in both groups. Only neurocognitive events were reported more frequently in the evolocumab group. Injection-site reactions were reported in 129 patients $(4.3 \%)$ in the evolocumab group.

\section{YUKAWA-2}

Yukawa-2 was a randomized, double-blind, placebocontrolled, phase III study evaluated the efficacy and safety of evolocumab in statin-treated Japanese patients at high cardiovascular risk, recently published in The American Journal of Cardiology in Jan. 2016. ${ }^{25}$

In total, 507 patients were screened for the study and 409 were randomized to $5 \mathrm{mg} /$ day or $20 \mathrm{mg}$ /day atorvastatin doses. Subsequently, patients underwent the second randomization to evolocumab 140mg biweekly (Q2W) or $420 \mathrm{mg}$ monthly (QM) or placebo Q2W or QM. The primary endpoint was percent reduction in LDLC levels from baseline to 12 weeks. Mean LDLC reductions at week 12 for evolocumab vs placebo ranged from $67 \%$ to 76\%. Efficacy and safety of Q2W or QM evolocumab dosing were similar.

\section{THOMAS-1 AND THOMAS-2}

Both studies were multicenter, open-label, randomized, parallel-arm, studies that enrolled patients at 22 and 23 sites (respectively) in the United States and Canada. ${ }^{26}$

Hypercholesterolemia or mixed dyslipidemia patients, 18-80 years of age, who were on statin therapy with or without ezetimibe, randomized to receive evolocumab administered at home with the prefilled evolocumab. Total number of three doses were administered over 6 weeks in THOMAS-1 (140mg Q2W) and 12 weeks in THOMAS-2 (420mg QM). The primary endpoint was the patient-reported successful outcome of attempted selfadministered full-dose of evolocumab in the home-use setting with the study device (autoinjector or PFS in THOMAS-1, autoinjector or AMD in THOMAS-2).

The secondary endpoint was the mean change in LDLC from baseline to 6 weeks in THOMAS-1 study and the mean of weeks 10 and 12 in THOMAS-2 study. In the THOMAS-1 study, 149 patients were randomized, and 144 completed the study (97\%). In the THOMAS-2 study, 164 were randomized, and 157 completed the study (96\%). Mean change (95\% CI) in THOMAS-1 was $63.4 \%$. In THOMAS-2, the mean change $(95 \% \mathrm{CI})$ was $64.5 \%$.

The most commonly occurring AEs in THOMAS-1 were a headache $(4 \%)$ bronchitis $(2 \%)$, and abdominal pain (2\%). The most commonly occurring AEs in THOMAS-2 were a pain in extremity, fatigue, and sinusitis (all 2\%). Total number of four patients discontinued the study because of AEs.

\section{FOURIER TRIAL}

Further Cardiovascular Outcomes Research with PCSK9 Inhibition in Subjects with Elevated Risk (FOURIER) was a double-blind, randomized, placebo-controlled, multicenter study (conducted at 1,272 sites across 49 countries) assessing the impact of additional LDLC reduction on major cardiovascular events when evolocumab is used in combination with statin therapy in patients with clinically evident cardiovascular disease. This study was completed in Nov 2016. Results from the FOURIER trial was published in the New England Journal of Medicine. ${ }^{27}$ There were 27,564 patients of age 40 to 85 years with fasting $\mathrm{LDLC} \geq 70 \mathrm{mg} / \mathrm{dL}$ $(\geq 1.8 \mathrm{mmol} / \mathrm{L})$ or non-HDLC $\geq 100 \mathrm{mg} / \mathrm{dL}(>2.6 \mathrm{mmol} / \mathrm{L})$ included in the trial. The first group received evolocumab Q2W or QM plus effective statin dose and the second group received placebo Q2W or QM plus effective statin dose. Results showed evolocumab reduced LDLC by 59 percent from a median of 92 to $30 \mathrm{mg} / \mathrm{dL}$, which remained steady throughout the duration of the study. There was 15 percent reduction in primary endpoints which were $\mathrm{CV}$ death, myocardial infarction (MI), stroke, and hospitalization for unstable angina or coronary revascularization. The trial confirmed $25 \%$ reduction in the serious secondary endpoint-cardiovascular death, heart attack or stroke-after the first year. The rate of adverse events, including muscle-related problems, allergic reactions, neurocognition impairment, and newonset diabetes was the same in both study arms. Only the rates of injection site reactions were more common with evolocumab as compared to placebo.

\section{GAUSS-3}

The aim of study was to identify patients with muscle symptoms caused by statin and compare lipid-lowering efficacy for 2 non-statin therapies, ezetimibe and evolocumab. Patients, 18-80 years of age, with elevated LDLC levels, who cannot tolerate statin because of muscle -related side effects, were enrolled in the GAUSS-3 trial. ${ }^{28}$ Co-primary end points were the mean percent change in LDLC level from baseline to the mean of weeks 22 and 24 levels and from baseline to week 24 levels. There were two phases of study, phase A in which atorvastatin $(20 \mathrm{mg}$ ) vs placebo was given and in phase B subcutaneous evolocumab (420mg monthly) or oral ezetimibe was given to the patients. 491 patients, who entered in phase A, muscle symptoms occurred in 209 $(42.6 \%)$ while taking atorvastatin but not while taking a placebo. Of 218 patients, assessed in phase B, 73 randomized to ezetimibe and 145 to evolocumab. Mean percent LDLC change, $-16.7 \%$ (95\% CI, $-20.5 \%$ to $12.9 \%$ ) in ezetimibe group and with evolocumab it was $54.5 \%$ (95\% CI, $-57.2 \%$ to $-51.8 \%$ ). In Ezetimibe-treated patients, muscle symptoms were reported in $28.8 \%$ and $20.7 \%$ in evolocumab- treated patients. 5 of 73 ezetimibetreated patients $(6.8 \%)$ and 1 of 145 evolocumab- treated patients $(0.7 \%)$ discontinued the study because of muscle symptoms. 


\section{THE GLAGOV TRIAL}

The GLAGOV trial was multicenter, international, double-blind, placebo- controlled, randomized clinical trial published in JAMA in Feb 2017. ${ }^{29}$ Total of 484 participants were randomized to the evolocumab group and 484 participants to the placebo group, and 423 participants in both groups completed the study. Patients were randomized to receive either subcutaneous injections of $420 \mathrm{mg}$ evolocumab monthly or placebo injections for 76 weeks. The primary outcome was the target artery change in percent atheroma volume (PAV) from baseline to week 78. Patients with statin and evolocumab not only achieved better stability of atherosclerotic plaque dimensions but actually results in regression of plaque size.

\section{DISCUSSION}

Statins are the first line drugs of the treatment for lowering LDLC concentration but additional LDLC lowering therapies are needed to reduce residual CV risk especially in patients at very high risk, statin intolerance or hereditary lipid disorders. PCSK9 inhibitors are the novel class of drugs in such patients. Promising data have come from recently published studies. Program to Reduce LDLC and cardiovascular Outcomes Following Inhibition of PCSK9 in different populations (PROFICIO), reflects LDL-C reduction via PCSK9 inhibition in both the clinic and at-home settings (Table 1). This program is composed of several completed studies that demonstrated safety and efficacy in hyperlipidemia and mixed dyslipidemia patients, as well as several ongoing studies that are evaluating safety and efficacy in the setting of atherosclerosis and secondary prevention.

Table 1: PROFICIO program.

\begin{tabular}{|ll|}
\hline Study & Aim \\
\hline GAUSS-2 & Statin intolerant patients \\
\hline GAUSS-3 & Statin intolerant patients \\
\hline $\begin{array}{l}\text { RUTHERFO } \\
\text { RD-2 }\end{array}$ & $\begin{array}{l}\text { Heterozygous Familial } \\
\text { hypercholesterolemia }\end{array}$ \\
\hline LAPLACE-2 & Combination therapy with statins \\
\hline MENDEL-2 & $\begin{array}{l}\text { Evolocumab stand-alone } \\
\text { monotherapy }\end{array}$ \\
\hline TESLA Part B & $\begin{array}{l}\text { Homozygous familial } \\
\text { hypercholesterolemia }\end{array}$ \\
\hline YUKAWA-2 & Combination therapy with statins \\
\hline DESCARTES & Long term (52 weeks) therapy \\
\hline FOURIER & Secondary prevention of CV events \\
\hline GLAGOV & $\begin{array}{l}\text { Plaque regression measured by } \\
\text { intravascular ultrasound (IVUS) }\end{array}$ \\
\hline THOMAS- I & Self-administered Evolocumab \\
\hline THOMAS- I & Self-administered Evolocumab \\
\hline
\end{tabular}

The addition of Evolocumab to existing lipid-lowering therapy has achieved significant (approximately 60\%) and the sustained reduction in LDLC levels in patients with varying levels of CV risk. Recently the results of FOURIER trial have shown that 15 percent reduction in CV death, MI, stroke, and hospitalization for unstable angina or coronary revascularization. Evolocumab has been found to be associated with lower incidences of adverse events. PCSK9 has a role in cortical neuron regeneration. Cholesterol is an important component of cell membrane and neurons. Excessive reduction of LDLC level may be associated with increased incidence of hormonal insufficiency, hemorrhagic stroke, neurocognitive impairment, and hemolytic anemia. ${ }^{30}$ Evaluating PCSK9 Binding antibody Influence on cognitive health in High cardiovascular Risk Subjects (EBBINGHAUS) study was a sub study of the FOURIER trial completed in Dec. 2016, found that the over a median follow-up of 19.8 months, there was no difference between patients in the evolocumab or placebo treatment groups, with respect to either primary or secondary endpoints. There was also no evidence to suggest differences in cognitive tests in patients attaining very low LDL cholesterol levels, including those with levels $<25 \mathrm{mg} / \mathrm{dl}$ or $0.65 \mathrm{mmol} / \mathrm{l}$.

Evolocumab has been approved by FDA in Aug. 2015. Results of FOURIER and GAUSS-3 were published after FDA approval of evolocumab.

\section{CONCLUSION}

Evolocumab has corroborated its effectiveness in reduction in the levels of LDLC. This drug has shown efficacy in heterozygous and homozygous subtypes of familial hypercholesterolemia. Statin intolerance seen in about $15 \%$ of all patients restricts the use of first line drug for dyslipidemia. Evolocumab can be a useful option in statin intolerant patients and in patients responding inadequately to statins.

Funding: No funding sources

Conflict of interest: None declared

Ethical approval: Not required

\section{REFERENCES}

1. Baigent C, Keech A, Kearney PM. Efficacy and safety of cholesterol-lowering treatment: prospective meta-analysis of data from 90,056 participants in 14 randomised trials of statins. Lancet. 2005;366:126778.

2. Cholesterol Treatment Trialists' (CTT) Collaboration. Efficacy and safety of more intensive lowering of LDL cholesterol: a meta-analysis of data from 170,000 participants in 26 randomised trials. Lancet. 2010;376:1670-81.

3. Cannon CP. Improved Reduction of Outcomes: vytorin Efficacy International Trial (IMPROVE-IT). Presented at the American Heart Association Scientific Sessions, Chicago; November 2014:15-18. 
4. Reiner Z, Catapano AL. ESC/EAS Guidelines for the management of dyslipidaemias The Task Force for the management of dyslipidaemias of the European Society of Cardiology (ESC) and the European Atherosclerosis Society (EAS). Eur. Heart J. 2011;32:1769-818.

5. Anderson TJ, Gregoire J. Update of the Canadian Cardiovascular Society Guidelines for the Diagnosis and Treatment of Dyslipidemia for the Prevention of Cardiovascular Disease in the Adult. Can. J. Cardiol. 2013;29:151-67.

6. Expert Panel on Detection, Evaluation, and Treatment of High Blood Cholesterol in Adults. Executive summary of the third report of the National Cholesterol Education Program (NCEP) Expert Panel on Detection, Evaluation, and Treatment of High Blood Cholesterol in Adults (Adult Treatment Panel III). JAMA. 2001;285(19):2486-97.

7. Cholesterol Treatment Trialists' (CTT) Collaborators, Mihaylova B, Emberson J. The effects of lowering LDL cholesterol with statin therapy in people at low risk of vascular disease: meta-analysis of individual data from 27 randomized trials. Lancet. 2012;380(9841):581-90.

8. Zhang H, Plutzky J, Skentzos S, Morrison F, Mar P, Shubina M, et al. Discontinuation of statins in routine care settings: a cohort study. Ann. Intern. Med. 2013;158(7):526-34.

9. Lambert G, Sjouke B, Choque B, Kastelein JJP, Hovingh GK. The PCSK9 decade. J. Lipid Res. 2012;53(12):2515-24.

10. Elguindy A, Yacoub MH. The discovery of PCSK9 inhibitors: A tale of creativity and multifaceted translational research. Glob. Cardiol. Sci. Pract. 2013;4:1-5.

11. Hong-mei G, Da-wei Z. Hypercholesterolemia, low density lipoprotein receptor and proprotein convertase subtilisin/kexin-type 9: The Journal of Biomedical Research. 2015;29(5):356-61.

12. Sawhney J, Bagga S. Proprotein convertase subtilisin kexin 9 inhibitors: Current status and future directions. J Clin Prev Cardiol. 2017;6:12-7.

13. Giugliano RP, Desai NR. Efficacy, safety, and tolerability of a monoclonal antibody to proprotein convertase subtilisin/kexin type 9 incombination with a statin in patients with hyper cholesterolaemia (LAPLACE-TIMI57): a randomised, placebocontrolled, dose- ranging, phase2 study. Lancet. 2012;380:2007-17.

14. Raal F, Scott R, Somaratne R, Bridges I, Li G, Wasserman SM, et al. Low-density lipoprotein cholesterol- lowering effects of AMG145, a monoclonal antibody to proprotein convertase subtilisin/ kexin type9 serine protease in patients with heterozygous familial hypercholesterolemia: the Reduction of LDL-C with PCSK9 Inhibi. Circulation. 2012;126:2408-17.

15. Sullivan D. Effect of a monoclonal antibody to PCSK9 on low-density lipoprotein cholesterol levels in statin-intolerant patients: The GAUSS randomized trial. JAMA. 2012;308:2497-506.

16. Stroes E. Anti-PCSK9 Antibody Effectively Lowers Cholesterol in Patients With Statin Intolerance: The GAUSS-2 Randomized, Placebo-Controlled Phase 3 Clinical Trial of Evolocumab. J Am Coll Cardiol. 2014;63(23):2541-8.

17. Koren MJ, Lundqvist P, Bolognese M. MENDEL-2 Investigators. Anti-PCSK9 monotherapy for hypercholesterolemia: the MENDEL-2 randomized, controlled phase III clinical trial of evolocumab. J Am Coll Cardiol. 2014;63(23):2531-40.

18. Robinson JG, Nedergaard B. LAPLACE-2 Investigators. Effect of evolocumab or ezetimibe added to moderate-or high-intensity statin therapy on LDLc lowering in patients with hypercholesterolemia: the LAPLACE-2 randomized clinical trial. JAMA. 2014;311(18):1870-82.

19. Bloom DJ, Hala T, Bolognese M. Descartes Investigators. A 52-week placebo-controlled trial of evolocumab in hyperlipidemia. $\mathrm{N}$ Engl $\mathrm{J}$ Med. 2014;370(19):1809-19.

20. Raal FJ, Honarpour N. Inhibition of PCSK9 with Evolocumab in homozygous familial hypercholesterolaemia (TESLA Part B): a randomised, double-blind, placebo-controlled trial. Lancet. 2015;385(9965):341-50.

21. Raal FJ, Stein EA. RUTHERFORD-2 Investigators. PCSK9 inhibition with evolocumab (AMG145) in heterozygous familial hypercholesterolemia (RUTHERFORD-2): a randomised, double-blind, placebo-controlled trial. Lancet. 2015;385(9965):331-40.

22. Sabatine MS, Giugliano RP. Investigators. Efficacy and Safety of Evolocumab in Reducing Lipids and Cardiovascular Events. N Engl J Med. 2015;372:1500-9.

23. Clinical Trials.gov. NCT01849497: a multi-center, randomized study in subjects with primary hypercholesterolemia or mixed dyslipidemia Available from: http://ClinicalTrials.gov/show/NCT01849497).

24. Clinical Trials.gov. NCT01879319: a randomized, multi-center clinical study in subjects with hypercholesterolemia or mixed dyslipidemia Available from: http://ClinicalTrials.gov/show/NCT01879319

25. Kiyosue A, Honarpour N. A Phase 3 Study of Evolocumab (AMG 145) in Statin-Treated Japanese Patients at High Cardiovascular Risk (YUKAWA-2): Am J Cardiol. 2016;117:40e47.

26. Dent. Evolocumab lowers LDL-C safely and effectively when self-administered in the at-home setting (THOMAS 1 and THOMAS 2): Springer Plus 2016;5:300.

27. Sabatine MS. Evolocumab and Clinical Outcomes in Patients with Cardiovascular Disease (FOURIER): N Engl J Med. 2017.

28. Nissen SE. Efficacy and Tolerability of Evolocumab vs Ezetimibe in Patients with Muscle-Related Statin 
Intolerance The (GAUSS-3): JAMA. 2016;315(15):1580-90.

29. Nicolls SJ, Puri S, Anderson, T. Effect of evolocumab on progression of coronary disease in statin-treated patients. The GLAGOV randomized clinical trial. JAMA. 2016;316:2372-84.
30. Dadu RT, Ballantyne CM. Lipid lowering with PCSK9 inhibitors. Nat. Rev. Cardiol. 2014;11(10):563-75.

Cite this article as: Mehani R, Shukla A, Yadav VK, Sahu R. Evolocumab: rising momentum as novel antidyslipidemic drug. Int $\mathrm{J}$ Basic Clin Pharmacol 2017;6:1538-44. 